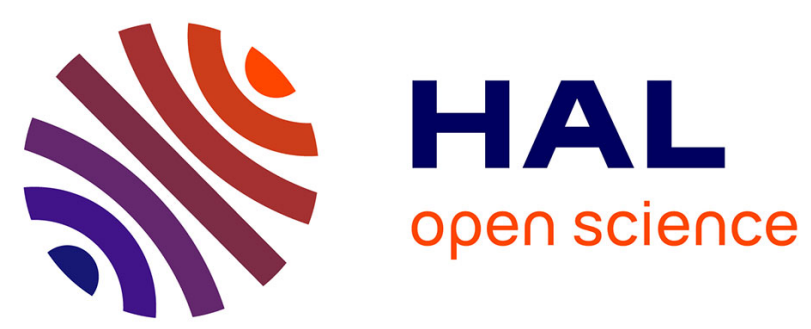

\title{
An Observer-based Longitudinal Control of Car-like Vehicles Platoon Navigating in an Urban Environment
}

Ahmed Khalifa, Olivier Kermorgant, Salvador Dominguez, Philippe Martinet

\section{To cite this version:}

Ahmed Khalifa, Olivier Kermorgant, Salvador Dominguez, Philippe Martinet. An Observer-based Longitudinal Control of Car-like Vehicles Platoon Navigating in an Urban Environment. CDC 2019 58th IEEE Conference on Decision and Control, Dec 2019, Nice, France. pp.5735-5741. hal-02273504

\section{HAL Id: hal-02273504 https://hal.science/hal-02273504}

Submitted on 29 Aug 2019

HAL is a multi-disciplinary open access archive for the deposit and dissemination of scientific research documents, whether they are published or not. The documents may come from teaching and research institutions in France or abroad, or from public or private research centers.
L'archive ouverte pluridisciplinaire HAL, est destinée au dépôt et à la diffusion de documents scientifiques de niveau recherche, publiés ou non, émanant des établissements d'enseignement et de recherche français ou étrangers, des laboratoires publics ou privés. 


\title{
An Observer-based Longitudinal Control of Car-like Vehicles Platoon Navigating in an Urban Environment
}

\author{
Ahmed Khalifa, Olivier Kermorgant, Salvador Dominguez and Philippe Martinet
}

\begin{abstract}
In this paper, we study longitudinal motion control of car-like vehicles platoon navigating in an urban environment with minimum communication links. To achieve a higher traffic flow, a constant-spacing policy between successive vehicles is commonly used but this is at a cost of an increased number of communication links as any vehicle information must broadcast to all its followers. Therefore, we propose a distributed observerbased control law that depends both on communicated and measured information. Our formulation allows designing the control law directly in the curvilinear coordinates. Internal and string stability analysis are conducted. We provide simulation results, through dynamic vehicular mobility simulator, to illustrate the feasibility of the proposed approach and corroborate our theoretical findings.
\end{abstract}

\section{INTRODUCTION}

Car sharing systems have attracted attention in recent years to overcome the urban transportation problems. However, cars redistribution among all stations is one of the main issues. One of the proposed solutions is to use vehicle platoons guided by professional drivers. A leader vehicle, driven by a human, comes to pick up the vehicles, then joins them to a platoon system, and finally drops off the vehicles over the stations so that the vehicles are well distributed.

In urban environments, the platoon motion has to be represented with respect to a path that the vehicle intends to follow. The curvilinear coordinates are usually used instead of absolute ones to represent the vehicle motion [1].

The global architecture of platoon control systems consists of two modules: longitudinal and lateral controllers. The former has two sub-modules including Upper-Level Controller (ULC), which provides the desired vehicle linear acceleration, and Lower-Level Controller (LLC), which generates the required engine throttle/brake to achieve this desired acceleration. Similarly, the lateral controller generates the required steering angle to achieve the desired lateral states. In this research, we are interested in designing the longitudinal ULC.

The framework of the longitudinal platoon control consists of four components, including Vehicle Dynamics, Information Flow Topology (IFT), Distributed Controller, and Spacing Policy [2].

For the vehicle dynamics, different models are used in the literature, including linear and nonlinear models [3]. Three linear models are frequently used including single integrator model [4], double integrator model [5], third order

The authors are with the Laboratoire des Sciences du Numérique de Nantes (LS2N), École Centrale de Nantes, France, firstname. namedec-nantes. fr.

P. Martinet is with Inria Sophia Antipolis, France. model [6]. Despite the huge amount of relevant literature to date, few studies in the literature have handled the problem of platooning in the urban environment/path coordinates, e.g. [7]. In these studies, the authors assume a first-order model for the vehicle dynamics which has many defects. In our previous work [8], [9], a second order longitudinal dynamic model is used and we have shown that one can design a controller to achieve control objectives in the path (operational) coordinates explicitly.

Three main platoon control objectives have to be achieved. First, the platoon can navigate in an urban environment (curvilinear path). Second, achieving position and velocity tracking for follower vehicles. Third, guarantee of the string stability, i.e., the error signals will attenuate when propagating downstream the vehicle string. The string instability may result in a rear-end collision between any predecessor/follower vehicles. In the literature, there are six main approaches in designing a platoon controller, including Linear [11], Optimal [12], $\mathcal{H}_{\infty}$-based [13], Sliding Mode [14], Model predictive [15], and Consensus-based controller [6]. In [8], [9], we present a consensus-based control algorithm that considers the motion of variable velocity platoon.

Several spacing policies are provided in the literature [16]. The constant distance spacing policy is used commonly due to its capability to achieve very high traffic capacity. However, to achieve string stability, more communication links are required to transmit leading vehicle information to all the following vehicles in the platoon.

The Information Flow Topology (IFT) represents the inter-vehicle communication topology that the vehicles use to acquire the information from its surrounding vehicles. The Predecessor-Leader Following (PLF) topology [16] is commonly used in the literature in which the leader communicates with all the vehicles in broadcast, and every vehicle also considers information from its predecessor to compute the control action. However, for either a low-cost onboard communication module that has limited bandwidth capabilities or for robust communication, high-speed twochannel communication is not always feasible or preferred. In this study, the topology assumption is called Hybrid PLF, in which only the leader broadcasts its information to all the vehicles. On the opposite, position or velocity information of a predecessor is not communicated but rather measured by its direct follower through a range sensor, e.g. laser. Therefore, we propose an observer-based control technique. A distributed longitudinal controller designed in the path coordinates is the basis of the proposed control strategy and its role is to achieve position and velocity consensus 
among the platoon members. An observer is then introduced in the loop to estimate the predecessor velocity. The merit of using the predecessor velocity in the control is twofold. It provides a faster convergence of the platoon with safety, and also results in a smoother acceleration of the motion of the vehicle.

In the literature, several observer-based consensus control algorithms are introduced for multi-agent systems in [17][19] and for platoon systems in [20]. However, these works use the observer to estimate the velocity of either the ego agent or the other vehicles by using the communication links among the agents. Moreover, they do not consider the string stability constraint during the controller design and analysis. In our study, the observer does not require any communication link with other vehicles/agents in the platoon which adds complexity in the design and analysis in order to guarantee both the platoon internal and string stability.

The main contributions of this paper are as follows:

- A distributed observer-based control algorithm is designed without the need to communicate with the preceding vehicle.

- The proposed algorithm can achieve higher traffic flow (by using constant spacing policy) with a minimum number of required communication links (by estimating the predecessor velocity with an observer).

- Conditions for both internal and string stability are provided.

- The proposed framework is validated via realistic simulation studies which are conducted in dynamic vehicular mobility simulator that considers real vehicle dynamics and platooning staff in an urban environment.

The rest of this paper is organized as follows. Section II formulates the platooning problem with kinematic and dynamic models expressed in both body and path coordinates. In Section III, the proposed control law is presented, and both internal and string stability are analyzed. Simulation studies are given in Section IV. Conclusions are made in Section V.

\section{Problem Statement}

\section{A. Vehicle Kinematic Model}

The kinematic model of a car-like vehicle can be simplified to that of a bicycle model [8], see Fig. 1.

1) Cartesian Coordinates: Let $\{V\}, O_{v}-x_{v} y_{v}$, represents the vehicle body-fixed reference frame with its $x$-axis in the vehicle's forward direction and its origin at the center of the rear axle, $O_{v}$. The configuration of the vehicle, with respect to the world-fixed inertial reference frame, $\{G\}, O-x y$, is represented by the generalized coordinates $[x, y, \theta]^{T} \in \mathbb{R}^{3}$, where its position is given by $[x, y]^{T}$ and its orientation in the global frame is represented by $\theta . \delta$ is the steering angle in the body frame. Since the wheels cannot slip sideways, the vehicle velocity is $v$ in the vehicle $x$-direction, and zero in the $y$-direction. Consider the distance $L$ from the rear wheel along the orientation of the vehicle to the front wheel. Then

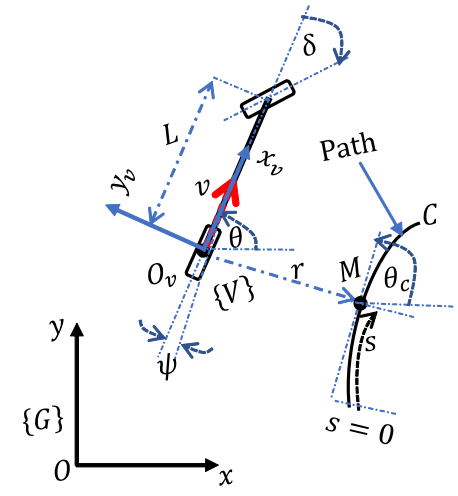

Fig. 1. Kinematic model of car-like vehicle.

the kinematic model is given by

$$
\begin{aligned}
\dot{x} & =v \cos (\theta), \\
\dot{y} & =v \sin (\theta), \\
\dot{\theta} & =\frac{v}{L} \tan (\delta)
\end{aligned}
$$

2) Path Coordinates Model: Let $[s, r, \psi]^{T}$ represents the curvilinear coordinates. The tracking path, $C$, defined in the Global Frame, can be represented as a function of several items including path length $s$ (curvilinear abscissa) at the closest point $M$ to $O_{v}$, the angular deviation, $\psi=\theta-\theta_{c}$, where $\theta_{c}$ is the angle between the path tangent at $M$ and the $x$-axis, and the lateral deviation, $r$, which is the signed orthogonal distance from the center of the rear axle, $O_{v}$, to the closest point on the path, $M$. Then, the kinematic model in the path coordinates is represented by

$$
\begin{aligned}
\dot{s} & =v \frac{\cos (\psi)}{1-r \kappa(s)}, \\
\dot{r} & =v \sin (\psi), \\
\dot{\psi} & =v\left(\frac{\tan (\delta)}{L}-\frac{\kappa(s) \cos (\psi)}{1-r \kappa(s)}\right)
\end{aligned}
$$

where $\kappa(s)$ is the path curvature at $M$.

\section{B. Car-like Vehicle Longitudinal Dynamic Model}

The vehicle longitudinal dynamic behavior is determined by several factors including the engine, drive line, brake system, aerodynamics drag, tire friction, gravitational force, etc [14]. For the vehicle model, we assume:

Assumption 1. The rigidity and symmetry of vehicle body.

Assumption 2. The integration of driving and braking inputs into one control input.

Assumption 3. The pitch and yaw motions effects are neglected.

The general longitudinal dynamic equation is given by

$$
M_{v} a+C_{v} v+G_{v}=F_{v}
$$

where $a=\dot{v}, M_{v}, C_{v}, G_{v}$ and $F_{v}$ denote the vehicle acceleration in the vehicle frame, the vehicle Inertia effect, Coriolis effect, Gravity effect and input force, respectively. 


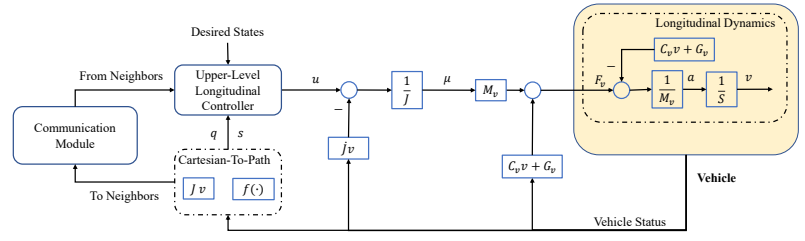

Fig. 2. Block diagram of the vehicle longitudinal dynamic model, where $S$ is the Laplace operator.

The longitudinal LLC being an inverse model compensation technique, it follows

$$
F_{v}=M_{v} \mu+C_{v} v+G_{v}
$$

where $\mu$ is theinput signal after system linearization which has to be designed. By applying this LLC, the second order dynamic model can be written in the body coordinates as

$$
a=\mu
$$

When platooning in an urban environment, the relation between the dynamics in the path and Cartesian coordinates will be derived such that the controller can be designed in the path coordinates. The block diagram of longitudinal controller with the dynamic model is given in Fig. 2.

To find a relation between the control signals in the path and body coordinates, let's recast (2a) as

$$
\dot{s}=J v
$$

where $J$ is given by

$$
J=\frac{\cos (\psi)}{(1-r \kappa)} .
$$

The acceleration in path coordinates, $\eta$, is thus given as

$$
\eta=J a+\dot{J} v
$$

where $\eta=\dot{q}=\ddot{s}$ and $q=\dot{s}$ are the vehicle acceleration and velocity in the path coordinates respectively. Consequently, if the mapping from the control signal (i.e., desired acceleration) in the path coordinates, $u$, to that in the body coordinates, $\mu$, is given by

$$
\mu=\frac{1}{J}(u-\dot{J} v),
$$

then, the vehicle longitudinal dynamic model in the path coordinates is given by

$$
\begin{aligned}
& \dot{s}=q, \\
& \dot{q}=\eta=u .
\end{aligned}
$$

\section{Platoon Longitudinal Model}

Consider a platoon of $N+1$ vehicles, as illustrated in Fig. 3 , including a leader (with index 0 ) and $N$ followers (with index $i ; i=1,2, \ldots, N)$. The vehicles can share information by either sensor-based or communication-based link. It is known that the vehicles involved in the car sharing system are often with the same type of small passenger vehicles, or have very close dynamics, hence we can assume

Assumption 4. All the vehicles have equal dynamics.

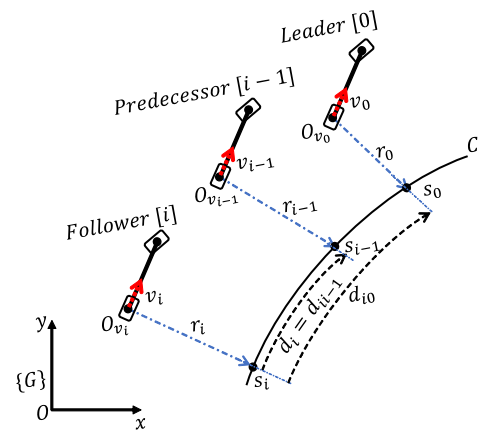

Fig. 3. Platoon of car-like vehicles.

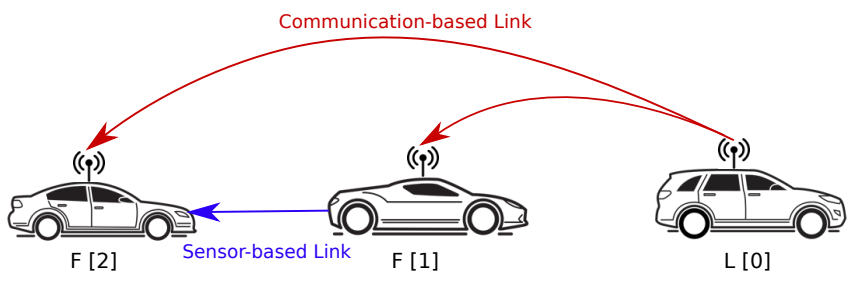

Fig. 4. Information flow topology: Hybrid PLF through communication link between leader and followers and direct sensor-based measurements between followers.

The in-platoon vehicle dynamics in the path coordinates can be given by

$$
\begin{aligned}
& \dot{s}_{i}=q_{i}, \\
& \dot{q}_{i}=\eta_{i}=u_{i},
\end{aligned}
$$

where the transformation between the two coordinates can be implemented using (9).

For the vehicles string, the inter-vehicle distance, $d_{i}=$ $s_{i-1}-s_{i}$, is the curvilinear distance between vehicle $i$ and its predecessor $i-1, d_{i 0}=s_{0}-s_{i}$, is the curvilinear distance between vehicle $i$ and leader, $d_{r, i}$ is the desired inter-vehicle distance between vehicles $i$ and $i-1$, and $d_{r, i 0}$ is the desired inter-vehicle distance between vehicles $i$ and 0 . For high traffic capacity, the constant spacing policy method is used, i.e. $d_{r, i}$ is constant.

\section{Control Objectives}

Under the hybrid IFT given in Fig. 4, the platoon is required to move in an urban environment (curvilinear path) and to track a variable velocity leader. Each follower $i$ can obtain leader information (i.e., position, velocity, and acceleration $\left.s_{0}, q_{0}, \eta_{0}\right)$ and predecessor position information (i.e., $\left.s_{i-1}\right)$. Under these circumstances, $\forall i=1, \ldots, N$, the following goals have to be achieved:

- estimation of the preceding vehicle velocity to be used in the distributed control law;

- convergence of the velocity estimation, position and velocity tracking errors;

- string stability.

\section{CONTROL DESIGN}

This section starts by presenting the proposed observerbased control algorithm. After that, the platoon internal 
stability analysis is presented. Finally, the string stability is analyzed.

\section{A. Observer-based Control Law}

The information flow structure among the platoon can be modeled by a graph in which the node dynamics are described by (11). The graph topology is described by an adjacency matrix $\mathcal{M}=\left[m_{i j}\right]_{N \times N}$, in which the element $m_{i j}=1$ if vehicle $i$ can obtain information from vehicle $j$; otherwise, $m_{i j}=0$. To include the leader (with index 0 ) in the network, an augmented graph is used. The Laplacian matrix $\mathbf{L}=\left[l_{i j}\right] \in \mathbb{R}^{N \times N}$ associated with the graph is given by $l_{i j}=-m_{i j}$ if $i \neq j$, and $l_{i j}=\sum_{k=1}^{N} m_{i k}$ if $i=j$.

Remark 1. For the Predecessor - Leader Following (PLF) information flow topology, the elements of the adjacency matrix is given as $m_{i j}=1$ if $j=i-1$, and $m_{i j}=0$ otherwise.

Let us consider a full PLF-based control law given as

$$
\begin{aligned}
u_{i}(t)= & \eta_{0}+g_{c, 2} e_{q, i 0}(t)+g_{c, 1} e_{s, i 0}(t) \\
& +g_{o, 1} e_{s, i}(t)+g_{o, 2} e_{q, i}(t),
\end{aligned}
$$

where $e_{s, i 0}(t)=s_{0}(t)-s_{i}(t)-d_{r, i 0}, e_{q, i 0}(t)=q_{0}(t)-q_{i}(t)$, $e_{s, i}(t)=s_{j}(t)-s_{i}(t)-d_{r, i}$, and $e_{q, i}(t)=q_{j}(t)-q_{i}(t) . G_{c}=$ $\left[g_{c, 1}, g_{c, 2}\right]^{T}, G_{o}=\left[g_{o, 1}, g_{o, 2}\right]^{T}$ are controller parameters to be designed.

Remark 2. In the proposed hybrid PLF, the information required to apply this control law (12) is available except the velocity of the predecessor or the velocity error between the ego vehicle $i$ and the predecessor velocity, $e_{q, i}$, which has to be estimated using an observer.

For observer design, let us define two states $z_{1, i}=e_{s, i}$ which is known (i.e., measured by the sensor mounted on vehicle $i$ ), and $z_{2, i}=e_{q, i}$ which is unknown and we need to estimate. The state equations that relates these two states are given by

$$
\begin{aligned}
& \dot{z}_{1, i}=z_{2, i}, \\
& \dot{z}_{2, i}=\dot{e}_{q, i} .
\end{aligned}
$$

The state $z_{2, i}$ can be estimated using the following observer

$$
\begin{aligned}
& \dot{\hat{z}}_{1, i}=\hat{z}_{2, i}+h_{1}\left(z_{1, i}-\hat{z}_{1, i}\right), \\
& \dot{\hat{z}}_{2, i}=h_{2}\left(z_{1, i}-\hat{z}_{1, i}\right) .
\end{aligned}
$$

Including the observer output in the control law (12), then the $i^{\text {th }}$ observer-based control law is proposed as

$$
\begin{aligned}
u_{i}(t)= & \eta_{0}+g_{c, 2} e_{q, i 0}(t)+g_{c, 1} e_{s, i 0}(t) \\
& +g_{o, 1} \hat{z}_{1, i}(t)+g_{o, 2} \hat{z}_{2, i}(t) .
\end{aligned}
$$

Let us define the error state vector of vehicle $i$ as $\mathbf{f}_{i}=$ $\left[f_{1, i}, f_{2, i}\right]^{T}$ with $f_{1, i}=e_{s, i 0}$ and $f_{2, i}=e_{q, i 0}$. Then, the $i^{\text {th }}$ closed-loop state space model is given by

$$
\dot{\mathbf{f}}_{i}(t)=\mathbf{A} \mathbf{f}_{i}(t)+\mathbf{B} \epsilon_{i}(t),
$$

where $\mathbf{A}=\left[\begin{array}{ll}0 & 1 \\ 0 & 0\end{array}\right], \mathbf{B}=\left[\begin{array}{l}0 \\ 1\end{array}\right]$, and

$$
\epsilon_{i}=-g_{c, 2} e_{q, i 0}-g_{c, 1} e_{s, i 0}-g_{o, 1} \hat{z}_{1, i}-g_{o, 2} \hat{z}_{2, i} .
$$

Exploiting that $z_{1, i}=e_{s, i}=e_{s, i 0}-e_{s, j 0}$ and let $\hat{\mathbf{z}}_{i}=$ $\left[\hat{z}_{1, i}, \hat{z}_{2, i}\right]^{T}$, then (14) can be rewritten concisely as

$$
\dot{\hat{\mathbf{z}}}_{i}=\mathbf{A}-\mathbf{H C} \hat{\mathbf{z}}_{i}+\mathbf{H C}\left(f_{1, i}-f_{1, j}\right)
$$

where $\mathbf{H}=\left[h_{1}, h_{2}\right]^{T}$ and $\mathbf{C}=[1,0,0]$.

Defining $\mathbf{G}_{c}=\frac{1}{2} \mathbf{K Q}_{1}, \mathbf{G}_{o}=\frac{1}{2} \mathbf{K Q}_{2}, \mathbf{K}=\left[k_{1}, k_{2}\right]$, and $\mathbf{Q}_{1}$ and $\mathbf{Q}_{2} \in \mathbb{R}^{2 \times 2}$, then (17) can be recast as

$$
\epsilon_{i}(t)=-\frac{1}{2} \mathbf{K} \mathbf{Q}_{1} \mathbf{f}_{i}(t)-\frac{1}{2} \mathbf{K Q}_{2} \hat{\mathbf{z}}_{i}(t),
$$

Let $\mathbf{f}=\left[\mathbf{f}_{1}^{T}, \ldots, \mathbf{f}_{N}^{T}\right]^{T}, \hat{\mathbf{z}}=\left[\hat{\mathbf{z}}_{1}^{T}, \ldots, \hat{\mathbf{z}}_{N}^{T}\right]^{T}$, then the system collective dynamics are given after some manipulations by:

$$
\begin{gathered}
\dot{\mathbf{f}}=\left(\mathbf{I}_{N} \otimes \mathbf{A}-\mathbf{I}_{N} \otimes \frac{1}{2} \mathbf{B K} \mathbf{Q}_{1}\right) \mathbf{f}-\left(\mathbf{I}_{N} \otimes \frac{1}{2} \mathbf{B K} \mathbf{Q}_{2}\right) \mathbf{z} \\
\dot{\hat{\mathbf{z}}}=\left(\mathbf{I}_{N} \otimes(\mathbf{A}-\mathbf{H C})\right) \hat{\mathbf{z}}+(\mathbf{D} \otimes \mathbf{H C}) \mathbf{f}
\end{gathered}
$$

where $\mathbf{I}_{N}$ denotes $(N \times N)$ identity matrix, $\mathbf{D}=\mathbf{L}+$ $\operatorname{diag}\{1,0,0\}$, and the operator $\otimes$ denotes Kronecker product.

\section{B. Internal Stability}

The closed loop system dynamics represented in (20) and (21) can be recast in the form

$$
\dot{\mathbf{X}}=\mathbf{A}_{c} \mathbf{X}
$$

where $\mathbf{X}=\left[\mathbf{f}^{T}, \mathbf{z}^{T}\right]^{T}$, and

$$
\mathbf{A}_{c}=\left[\begin{array}{cc}
\mathbf{I}_{N} \otimes\left(\mathbf{A}-\frac{1}{2} \mathbf{B K} \mathbf{Q}_{1}\right) & -\mathbf{I}_{N} \otimes \frac{1}{2} \mathbf{B K} \mathbf{Q}_{2} \\
\mathbf{D} \otimes \mathbf{H} \mathbf{C} & \mathbf{I}_{N} \otimes(\mathbf{A}-\mathbf{H C})
\end{array}\right]
$$

For the internal stability of the proposed platoon observerbased control system let us present the following theorem.

Theorem III.1. Consider the distributed control of a platoon of connected car-like vehicles with dynamics defined in (2), (3) - (9), and (11), with assumptions 1 - 4, and the observerbased control algorithm proposed in (14) and (15) is applied to it. Then, the system is asymptotically stable as long as the following conditions are met.

1) Find $\Gamma \in \mathbb{R}^{2 \times 2}$ to satisfy

$$
(\mathbf{A}-\mathbf{H C}) \boldsymbol{\Gamma}-\boldsymbol{\Gamma}(\mathbf{A}-\mathbf{B K})=-\lambda_{i} \mathbf{H C}
$$

where $\lambda_{i}$ is the $i^{\text {th }}$ eigenvalue of the matrix $\mathbf{D}$.

2) The gain matrices $\mathbf{Q}_{1}$ and $\mathbf{Q}_{2}$ must satisfy

$$
\left[\frac{1}{2} \mathbf{Q}_{1}, \frac{1}{2} \mathbf{Q}_{2}\right]=\left[\begin{array}{c}
\mathbf{I}_{2} \\
\mathbf{\Gamma}
\end{array}\right]^{-1}
$$

3) Constraints on the gain vector $\mathbf{K}$ are

$$
k_{1}>0 \text { and } k_{2}>0
$$

4)

$$
\lambda_{\max }\left\{\frac{1}{2} \mathbf{\Gamma B K Q}_{2}\right\}<\lambda_{\min }\{-(\mathbf{A}-\mathbf{H C})\}
$$

Proof. For the PLF information flow topology, D is an upper-triangular matrix and its eigenvalues, $\lambda_{i}$, are real. 
Therefore, for vehicle $i$, the matrix $\mathbf{A}_{c}$ can be represented as

$$
\overline{\mathbf{A}}_{c}=\left[\begin{array}{cc}
\mathbf{A}-\frac{1}{2} \mathbf{B K Q _ { 1 }} & -\frac{1}{2} \mathbf{B K \mathbf { Q } _ { 2 }} \\
\lambda_{i} \mathbf{H C} & \mathbf{A}-\mathbf{H C}
\end{array}\right]
$$

Exploiting the similarity transformation technique by multiplying the left and right sides of the matrix $\overline{\mathbf{A}}_{c}$ by $\overline{\mathbf{Q}}=$ $\left[\begin{array}{cc}\mathbf{I} & 0 \\ -\boldsymbol{\Gamma} & \mathbf{I}\end{array}\right]$, and $\overline{\mathbf{Q}}^{-1}=\left[\begin{array}{ll}\mathbf{I} & 0 \\ \boldsymbol{\Gamma} & \mathbf{I}\end{array}\right]$, respectively, and by conditions (24) and (25), one can obtain

$$
\overline{\mathbf{Q}} \overline{\mathbf{A}}_{c} \overline{\mathbf{Q}}^{-1}=\left[\begin{array}{cc}
\mathbf{A}-\mathbf{B K} & -\frac{1}{2} \mathbf{B K} \mathbf{Q}_{2} \\
0 & \mathbf{A}-\mathbf{H C}+\frac{1}{2} \mathbf{\Gamma B K} \mathbf{Q}_{2}
\end{array}\right]
$$

Therefore, $\quad \operatorname{eig}\left\{\overline{\mathbf{A}}_{c}\right\} \quad=\quad \operatorname{eig}\{\mathbf{A}-\mathbf{B K}\} \cup$ $e i g\left\{\mathbf{A}-\mathbf{H C}+\frac{1}{2} \mathbf{\Gamma} \mathbf{B K} \mathbf{Q}_{2}\right\}$, where $\operatorname{eig}\{\mathbf{M}\}$ denotes the eigenvalues of $\mathbf{M}$. To ensure $\left(\mathbf{A}-\mathbf{H C}+\frac{1}{2} \boldsymbol{\Gamma B K Q} \mathbf{Q}_{2}\right)<0$, condition (27) must be satisfied. Moreover, the characteristic equation of the matrix $(\mathbf{A}-\mathbf{B K})$ can be expressed as

$$
\operatorname{det}\left(S \mathbf{I}_{2}-(\mathbf{A}-\mathbf{B K})\right)=S^{2}+k_{2} S+k_{1}
$$

According to Routh-Hurwitz stability criterion [21], this polynomial (30) is Hurwitz stable under condition (26). This implies that all eigenvalues of $\overline{\mathbf{A}}_{c}$ and consequently $\mathbf{A}_{c}$ have negative real parts.

Remark 3. Adding observer to the control loop increases the complexity of the string stability analysis. Therefore, the next corollary is proposed in order to reduce the number of design parameters which in turn simplifies both the observer-based control design and string stability analysis.

Corollary III.2. The proposed platoon observer-based control system is asymptotically stable provided that the design parameters are selected as

$$
\begin{aligned}
& k_{1}=p_{c}^{2}, \\
& k_{2}=2 p_{c}, \\
& h_{1}=2 p_{o}, \\
& h_{2}=p_{o}^{2}, \\
& p_{o}=\gamma p_{c}, \forall \gamma, p_{c}, \text { and } p_{o}>0, \\
& \gamma>\frac{1}{2} \text { and } \gamma \neq 1,
\end{aligned}
$$

where the desired eigenvalues of controller part (A-BK) are assigned at $-p_{c}$, and that of the observer part, (A $\mathbf{H C )}$, are placed at $-p_{o} . \gamma$ is a constant that determines the distance between the poles of the controller and the observer.

Proof. By choosing $\mathbf{K}$ and $\mathbf{H}$ as given in conditions (31a) (31d), all the poles of $(\mathbf{A}-\mathbf{B K})$ and $(\mathbf{A}-\mathbf{H C})$ are placed at $-p_{c}$ and $-p_{o}$ respectively. Hence, one can choose the eigenvalues to be different by satisfying (31f). Thus, solution of (24), $\boldsymbol{\Gamma}$, exists, and consequently, condition (25) is met.

Checking stability conditions of (30), and setting the value of $k_{1}$ and $k_{2}$, as given in (31a) - (31b), we can satisfy condition (26).
The design of the algorithm parameters as in (31a) - (31e) has another merit that is the number of the design parameters are reduced to $\gamma$ and $p_{c}$.

Substitution (31a) - (31e) into (27), after some algebraic manipulations, the roots of $\left(\mathbf{A}-\mathbf{H C}+\frac{1}{2} \mathbf{\Gamma} \mathbf{B K} \mathbf{Q}_{2}\right)$ are $\left(\frac{1}{2}-r \pm \frac{1}{2} \sqrt{4 r-5}\right)$ which are negative definite under a condition for $\gamma$ given in (31f).

\section{String Stability}

In addition to internal stability, the platoon controller has to guarantee string stability; otherwise, the error signals will amplify when propagating downstream the vehicle string, which may result in rear-end collision.

Definition III.3. $L_{2}$ String Stability means that the energy (represented by the $L_{2}$ norm) of the position error of vehicle $i, e_{s, i}$, is smaller than the energy of the position error of proceeding vehicle $j=i-1, e_{s, j}$ [22]. Since $\left\|\bar{G}(S)=\frac{E_{i}(S)}{E_{j}(S)}\right\|_{\infty}=\sup _{e_{s, j} \in L_{2}} \frac{\left\|e_{s, i}\right\|_{2}}{\left\|e_{s, j}\right\|_{2}}$, the string stability is guaranteed if

$$
\|\bar{G}(S)\|_{\infty}<1
$$

For simplicity of notation and analysis, let us define the following errors $e_{i}(t)=s_{j}(t)-s_{i}(t)-d_{r, i}, \dot{e}_{i}(t)=q_{j}(t)-$ $q_{i}(t), e_{i 0}(t)=s_{0}(t)-s_{i}(t)-d_{r, i 0}$, and $\dot{e}_{i 0}(t)=q_{0}(t)-q_{i}(t)$.

Theorem III.4. Consider the platoon system presented in Theorem III.1 and the observer-based control algorithm proposed in (15) and its parameterization given in Corollary III.2. Then, the platoon is string stable as long as the conditions (31a) - (31e) are satisfied and the choice of factor $\gamma$ is constrained by

$$
\gamma \geq \sqrt{\frac{9}{12}}
$$

Proof. Let us start with

$$
\ddot{e}_{i}=\eta_{j}-\eta_{i}
$$

Substituting (11) and (15) into (34), considering the relations $e_{i}=e_{i 0}-e_{j 0}$ and $\dot{e}_{i}=\dot{e}_{i 0}-\dot{e}_{j 0}$, and after some algebraic manipulations, we get

$\ddot{e}_{i}=-g_{c, 2} \dot{e}_{i}-g_{c, 1} e_{i}-g_{o, 1} \hat{z}_{1, i}-g_{o, 2} \hat{z}_{2, i}+g_{o, 1} \hat{z}_{1, j}+g_{o, 2} \hat{z}_{2, j}$.

The observer model given (18) can be represented in the form of transfer functions as

$$
\begin{aligned}
& \bar{G}_{z_{1}}(S)=\frac{\hat{Z}_{1, i}(S)}{E_{j}(S)}=\frac{h_{1} S+h_{2}}{S^{2}+h_{1} S+h_{2}}, \\
& \bar{G}_{z_{2}}(S)=\frac{\hat{Z}_{2, i}(S)}{E_{j}(S)}=\frac{h_{2} S}{S^{2}+h_{1} S+h_{2}} .
\end{aligned}
$$

Writing (35) in the S-domain along with (36) yields:

$$
\bar{G}(S)=\frac{g_{o, 1} \bar{G}_{z_{1}}+g_{o, 2} \bar{G}_{z_{2}}}{S^{2}+g_{c, 2} S+g_{c, 1}+g_{o, 1} \bar{G}_{z_{1}}+g_{o, 2} \bar{G}_{z_{2}}} .
$$


As $\|\bar{G}(S)\|_{\infty}=\sup _{w>0} \sqrt{|\bar{G}(j w)|^{2}}=\sup _{w>0} \sqrt{\frac{\bar{G}_{n}(w)}{\bar{G}_{d}(w)}}$, string stability criterion (32) is equivalent to

$$
\bar{G}_{d}(w)-\bar{G}_{n}(w)>0, \forall w>0 .
$$

Exploiting the definitions of $G_{c}$ and $G_{o}$ along with (31a) (31e), after certain algebraic manipulations, (38) is recast as

$$
\bar{G}_{d}(w)-\bar{G}_{n}(w)=4 w^{8}+a_{6} w^{6}+a_{4} w^{4}+a_{2} w^{2}+a_{0},
$$

where coefficients $a_{(.)}$are functions of $\gamma$ and $p_{c}$ :

$$
\begin{aligned}
& a_{6}=8 \gamma^{2} p_{c}^{2}, \\
& a_{4}=-p_{c}^{4}\left(-4 \gamma^{4}+16 \gamma^{3}-44 \gamma^{2}+24 \gamma+3\right), \\
& a_{2}=2 p_{c}^{6}\left(2 \gamma^{4}-4 \gamma^{3}+6 \gamma^{2}-3\right), \\
& a_{0}=\gamma^{2} p_{c}^{8}\left(3 \gamma^{2}-\gamma+6\right) .
\end{aligned}
$$

After some algebraic and numeric manipulations, under condition (33), we find that all the coefficients $a_{6}-a_{0}$ are positive definite independently from the value of $p_{c}$, and hence, the string stability constraint (38) is satisfied.

\section{RESULTS AND DISCUSSION}

\section{A. Study Setup}

The proposed control strategy is tested by implementing the vehicles platoon in a custom simulator called ICARS that is developed under the Robot Operating System (ROS). ICARS has features such as real vehicle dynamics and models, both low and high level measurement units with noises, etc. The platoon is tested in a virtual map of Centrale Nantes (ECN) campus as an example of urban environment (road with curvatures, bumps, humans, etc).

We consider a platoon composed of $N$ follower vehicles (of type Renault ZOE) plus a manually driven leader (of type Renault FLUENCE). The physical parameters of the vehicles are given in Table I.

The vehicles are initially parked. While the leader moves to the destination points, its path is built online and sent via the radio communication module to the followers as the reference path to follow. The radio system receives the vehicle information in the platoon one by one.

The lateral controller presented in [23] is in charge of producing the steering angle of the vehicle to track the reference path of the leader (by minimizing both the lateral and angular deviations, $r_{i}$ and $\psi_{i}$, respectively).

The longitudinal controller is implemented at a rate of $100 \mathrm{~Hz}$. The control algorithm parameters are selected to guarantee the internal stability and string stability given in Theorem III.1, Corollary III.2 and Theorem III.4. Table II presented the control parameters. For safety and comfortability, the vehicle velocity and acceleration are constrained to certain levels as given in Table II.

\section{B. Simulation Results}

Three Renault ZOE are used as followers, i.e., $N=3$. Lateral motion is illustrated in Fig. 5a - Fig. 5c. The reference and actual paths of the leader and followers respectively are shown in Fig. 5a. Fig. 5b and 5c present lateral and angular deviations of the followers with respect to the reference
TABLE I

VEHICLES PHYSICAL PARAMETERS

\begin{tabular}{|c|c|c|}
\hline Parameter/Vehicle & FLUENCE & ZOE \\
\hline Mass & $1605 \mathrm{~kg}$ & $1428 \mathrm{~kg}$ \\
\hline Width & $1.545 \mathrm{~m}$ & $1.945 \mathrm{~m}$ \\
\hline Height & $1.462 \mathrm{~m}$ & $1.562 \mathrm{~m}$ \\
\hline Length & $4.748 \mathrm{~m}$ & $4.084 \mathrm{~m}$ \\
\hline Rear-axis to Rear-end & $1.140 \mathrm{~m}$ & $0.657 \mathrm{~m}$ \\
\hline distance between axes & $2.701 \mathrm{~m}$ & $2.588 \mathrm{~m}$ \\
\hline distance between wheels & $1.545 \mathrm{~m}$ & $1.511 \mathrm{~m}$ \\
\hline Wheel radius & $0.29 \mathrm{~m}$ & $0.29 \mathrm{~m}$ \\
\hline Inertia momentum & $45.0 \mathrm{kgm}^{2}$ & $28.0 \mathrm{kgm}^{2}$ \\
\hline
\end{tabular}

TABLE II

CONTROLLER PARAMETERS

\begin{tabular}{|c|c|c|c|}
\hline Parameter & Value & Parameter & Value \\
\hline$u_{i}$ & $\in[-6,1] \mathrm{m} / \mathrm{s}^{2}$ & $v_{i}$ & $\in[0,8] \mathrm{m} / \mathrm{s}$ \\
\hline$d_{r, i}$ & $10 \mathrm{~m}$ & $\gamma$ & 6 \\
\hline$p_{c}$ & 1 & $\lambda_{i}$ & 1 \\
\hline
\end{tabular}

path generated form the leader. Initial high deviations due to the time taken by the followers to join the platoon and converge to the leader path. The deviations increase when the leader moves through a curved segment of the road/path. These figures enlighten the tracking capabilities of the lateral controller and the potential of the longitudinal one.

Fig. $5 d-5 h$ show the longitudinal motion results. As shown in Fig. 5e, the leader moves with variable velocity depending on the path conditions (by a human driver). This study investigates also the effect of accelerations perturbations as shown in Fig. 5d. The vehicles eventually reach the consensus. At certain places in the path (e.g., road curvature), the position and velocity errors become higher which is desired to allow the follower to adapt its velocity based on the road conditions. The observer estimation error, $\tilde{z}_{2, i}=e_{q, i j}-\hat{z}_{2, i}$, is given in Fig. 5 h which shows a convergence of the estimated predecessor velocity.

To quantify our results, the Root Mean Squared Error (RMSE) of the estimation and tracking errors, calculated after the initialization period is passed, are reported in Table III which are in the acceptable ranges. Furthermore, this table shows that the position tracking errors are attenuated as the RMSE of $e_{s, 3}<e_{s, 2}<e_{s, 1}$, which illustrate the string stability of the platoon.

TABLE III

RMSE OF TRACKING ERRORS IN SIMULATION.

\begin{tabular}{|c|c|c|c|}
\hline RMSE Follower $i$ & 1 & 2 & 3 \\
\hline$e_{s, i}[\mathrm{~m}]$ & 0.2675 & 0.1141 & 0.0290 \\
\hline$e_{q, i}[\mathrm{~m} / \mathrm{s}]$ & 0.1199 & 0.0635 & 0.0480 \\
\hline$\tilde{z}_{2, i}[\mathrm{~m} / \mathrm{s}]$ & - & 0.0635 & 0.0480 \\
\hline$r_{i}[\mathrm{~m}]$ & 0.1324 & 0.1080 & 0.1104 \\
\hline$\psi_{i}[\mathrm{rad}]$ & 0.0209 & 0.0196 & 0.0198 \\
\hline
\end{tabular}

Demonstration of this study is available in this video ${ }^{1}$.

\footnotetext{
${ }^{1}$ https://www.dropbox.com/s/te3p0dod0kjtye3/obsrv_ sim.mp 4 ? dl=0
} 


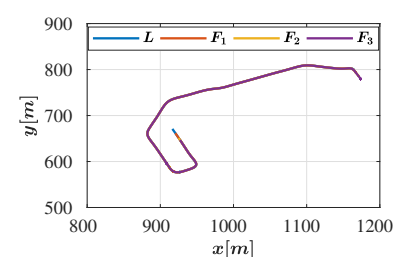

(a) Path tracking: path

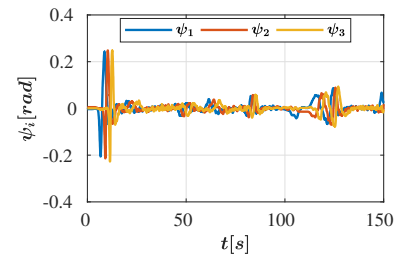

(c) Path tracking: orientation error

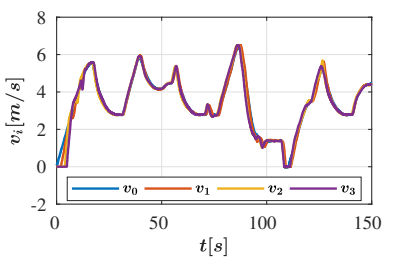

(e) Vehicles travel with variable velocity

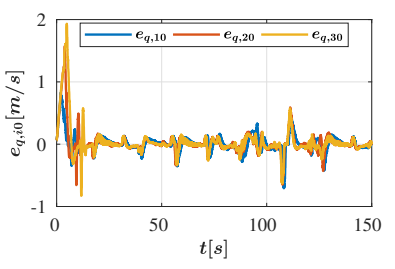

(g) Velocity tracking

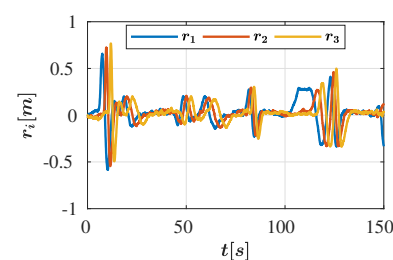

(b) Path tracking: lateral error

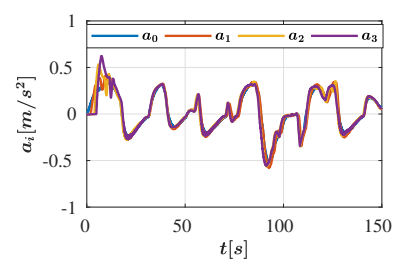

(d) Acceleration perturbation

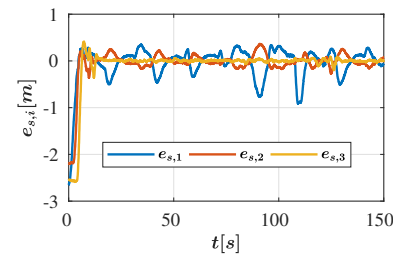

(f) Position tracking

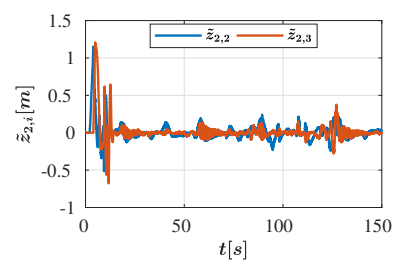

(h) $\tilde{z}_{2, i}=e_{q, i j}-\hat{z}_{2, i}$
Fig. 5. Platoon simulation results. $L$ : leader actual path, and $F_{i}$ : follower $i$ actual path. The followers track the path perfectly

\section{CONCLusions}

Platoon kinematic and dynamic models are introduced in both curvilinear and Cartesian coordinates with a framework that allows us to design the control law in the curvilinear coordinates. An observer-based control law is proposed to achieve both consensus and string stability of the platoon vehicles. This control scheme requires a minimum number of communication links and can still achieve a higher traffic flow. This strategy reduces the traffic in the communication channel which in turn can enhance the communication robustness, and enables us to use a low-cost communication module. Conditions for platoon internal stability and string stability are provided. Simulation studies are conducted and illustrate the relevance of our approach.

\section{ACKNOWLEDGEMENTS}

The work presented in this paper is carried out in the framework of the Valet project, reference ANR-15-CE220013-02.

\section{REFERENCES}

[1] T. D. Barfoot and C. M. Clark, "Motion planning for formations of mobile robots," Robotics and Autonomous Systems, 2004.

[2] S. E. Li, Y. Zheng, K. Li, and J. Wang, "An overview of vehicular platoon control under the four-component framework," in IEEE Intelligent Vehicles Symposium (IV), 2015.

[3] W. Yue, G. Guo, L. Wang, and W. Wang, "Nonlinear platoon control of arduino cars with range-limited sensors," Int. Journal of Control, 2015.

[4] F. Lin, M. Fardad, and M. R. Jovanovic, "Optimal control of vehicular formations with nearest neighbor interactions," IEEE Transactions on Automatic Control, 2012.

[5] R. Lemos, O. Garcia, and J. V. Ferreira, "Local and global path generation for autonomous vehicles using splines," Ingeniería, 2016.

[6] J. C. Zegers, E. Semsar-Kazerooni, J. Ploeg, N. van de Wouw, and H. Nijmeijer, "Consensus control for vehicular platooning with velocity constraints," IEEE Trans. on Control Systems Technology, 2017.

[7] P. Avanzini, B. Thuilot, and P. Martinet, "A control strategy taking advantage of inter-vehicle communication for platooning navigation in urban environment," in IROS11 Int. workshop on Perception and Navigation for Autonomous Vehicles in Human Environment, 2011.

[8] A. Khalifa, O. Kermorgant, S. Dominguez, and P. Martinet, "Vehicles platooning in urban environment: Consensus-based longitudinal control with limited communications capabilities," in Int. Conf. on Control, Automation, Robotics and Vision, 2018.

[9] — - "Vehicles platooning in urban environments: Integrated consensus-based longitudinal control with gap closure maneuvering and collision avoidance capabilities," in European Control Conf., 2019.

[10] J. VanderWerf, S. Shladover, N. Kourjanskaia, M. Miller, and H. Krishnan, "Modeling effects of driver control assistance systems on traffic," Transportation Research Record, 2001.

[11] H. Chehardoli and M. R. Homaeinezhad, "Third-order leaderfollowing consensus protocol of traffic flow formed by cooperative vehicular platoons by considering time delay: constant spacing strategy," the Journal of Systems and Control Engineering, 2018.

[12] M. Fardad, F. Lin, and M. R. Jovanović, "Sparsity-promoting optimal control for a class of distributed systems," in IEEE American Control Conf. (ACC), 2011.

[13] F. Gao, S. E. Li, Y. Zheng, and D. Kum, "Robust control of heterogeneous vehicular platoon with uncertain dynamics and communication delay," IET Intelligent Transport Systems, 2016.

[14] Y. Wu, S. E. Li, Y. Zheng, and J. K. Hedrick, "Distributed sliding mode control for multi-vehicle systems with positive definite topologies," in IEEE Conf. on Decision and Control (CDC), 2016.

[15] W. B. Dunbar and D. S. Caveney, "Distributed receding horizon control of vehicle platoons: Stability and string stability," IEEE Transactions on Automatic Control, 2012.

[16] D. Swaroop, J. Hedrick, C. Chien, and P. Ioannou, "A comparision of spacing and headway control laws for automatically controlled vehicles1," Vehicle System Dynamics, 1994.

[17] C. Wen, F. Liu, Q. Song, and X. Feng, "Observer-based consensus of second-order multi-agent systems without velocity measurements," Neurocomputing, 2016.

[18] L. Gao, B. Xu, J. Li, and H. Zhang, "Distributed reduced-order observer-based approach to consensus problems for linear multi-agent systems," IET Control Theory \& Applications, 2015.

[19] D. Ding, Z. Wang, D. W. Ho, and G. Wei, "Observer-based eventtriggering consensus control for multiagent systems with lossy sensors and cyber-attacks," IEEE transactions on cybernetics, 2017.

[20] M. Yan, Y. Tang, P. Yang, and L. Zuo, "Consensus based platoon algorithm for velocity-measurement-absent vehicles with actuator saturation," Journal of Advanced Transportation, 2017.

[21] R. C. Dorf and R. H. Bishop, Modern control systems. Pearson, 2011.

[22] J. Eyre, D. Yanakiev, and I. Kanellakopoulos, "A simplified framework for string stability analysis of automated vehicles ", Vehicle System Dynamics, 1998.

[23] B. Thuilot, J. Bom, F. Marmoiton, and P. Martinet, "Accurate automatic guidance of an urban electric vehicle relying on a kinematic gps sensor," IFAC Proceedings Volumes, 2004. 